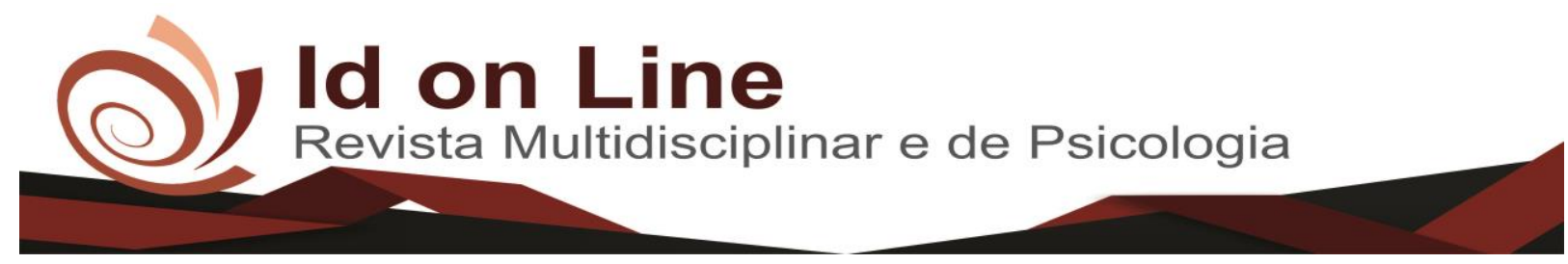

Artigo

\title{
Estresse Laboral em Profissionais da Saúde na Ambiência da Unidade de Terapia Intensiva
}

\author{
Antonio Taumaturgo Sampaio Macêdo ${ }^{1}$, Morgana Tavares Dantas Sousa ${ }^{2}$, Rodrigo Luís Mousinho Gomes ${ }^{3}$, \\ Marco Antônio Bezerra Rolim ${ }^{4}$, Jussara Evila Pinheiro Basto ${ }^{5}$, Renata da Silva Adonias Dantas ${ }^{6}$, \\ Hermes Melo Teixeira Batista ${ }^{7}$ : Eliane de Sousa Leite ${ }^{8}$
}

\begin{abstract}
Resumo: O trabalho pode gerar sofrimento ao trabalhador dependendo da forma que é vivido, manifestando-se na forma de estresse, caracterizado pela readaptação do organismo a um agente que está causando algum desequilíbrio. Este estudo teve como objetivo principal investigar os fatores associados ao estresse laboral em profissionais que trabalham em ambiente de Unidade de Terapia Intensiva. Para tanto, foi realizado um estudo observacional, no qual médicos, fisioterapeutas e equipes de enfermagem de 02 (dois) hospitais da cidade de Barbalha, Ceará, responderam aos questionários sociodemográfico MTEG (modelo teórico para explicar o estresse em gerentes) e O Inventário de Sintomas de Stress para adultos de Lipp (ISSL). Participaram da pesquisa 55 profissionais, sendo os dados coletados em dois momentos: No primeiro os questionários foram entregues dentro de um envelope e no segundo os termos foram preenchidos junto ao aplicador, de maneira a evitar maiores constrangimentos. Ao final do trabalho pôde-se concluir que a maior prevalência de sintomas de estresse se deu nos profissionais da classe dos fisioterapeutas, do sexo feminino, com idade menor do que 38 anos e com menos de 4 anos de profissão. Os dados também revelam que, dos 55 indivíduos pesquisados, 16 (29\%) apresentaram critérios positivos para estresse laboral, sendo 1 (2\%)na fase 1 ou de alerta ou alarme, $13(24 \%)$ na fase 2 ou luta ou resistência e $2(3 \%)$ na fase 3 ou exaustão ou esgotamento. Quanto aos sintomas de estresse manifestados destacaram-se:Tensão muscular $(81 \%)$ - nas últimas 24 horas, problema com a memória, esquecimento $(81 \%)$ e sensação de desgaste físico constante $(81 \%)$ - no último mês, cansaço excessivo $(75 \%)$ e angústia ou ansiedade diária $(69 \%)$ - nos últimos 3 meses.Propõe-se uma reflexão sobre possíveis medidas a serem adotadas no sentido de melhorar as condições de trabalho e sugerimos a realização de estudos mais elaborados sobre as repercussões do estresse com ênfase no profissional de Unidade de Terapia Intensiva.
\end{abstract}

Palavras-chave: Estresse laboral. Esgotamento profissional.Estresse ocupacional.Profissionais de saúde. UTI

\section{Labor Stress in Health Professionals in the ambience of the Intensive Care Unit}

\begin{abstract}
The work can generate suffering to the worker depending on the form that is lived, manifesting itself in the form of stress, characterized by the adaptation of the organism to an agent that is causing some imbalance.The main objective of this study was to investigate the factors associated with work stress in professionals working in an Intensive Care Unit. An observational study was conducted in which physicians, physiotherapists and nursing teams from 02 (two) hospitals in the city of Barbalha, Ceará, answered the MTEG sociodemographic questionnaire
\end{abstract}

\footnotetext{
${ }^{1}$ Graduado em fisioterapia pela Faculdade de Medicina Estácio FMJ - Estácio FMJ. E-mail: taumaturgomacedo@yahoo.com;

${ }^{2}$ Graduada em medicina pela Faculdade de Medicina Estácio FMJ - Estácio FMJ. E-mail: morgtds@ gmail.com;

${ }^{3}$ Mestrado em Medicina Interna pela Universidade Federal de Pernambuco, Brasil;

${ }^{4}$ Mestrando em Ciências da Saúde pela FMABC

${ }^{5}$ Enfermeira do Hospital Universitário Júlio Bandeira - HUJB, Cajazeiras, PB. Contato: Jussara.bastos@ebserh.gov.br

${ }^{6}$ Mestre em Ciências da Saúde pela FMABC.

${ }^{7}$ Doutorando em Ciências da Saúde pela FMABC, Médico do Hospital Universitário Júlio Bandeira - HUJB, Cajazeiras, PB, Professor de Emergências médica da Estácio, FMJ. Contato: hermesmelo@oi.com.br;

${ }^{8}$ Doutorado em Enfermagem pela Universidade Federal da Paraíba, Brasil. Chefe Setor de Apoio Diagnóstico Terapêutico do Hospital Universitário Júlio Bandeira, Brasil.
} 
(theoretical model to explain stress in managers) and the Inventory of Symptoms of Stress for Adults of Lipp (ISSL). Fifty-five professionals participated in the study, and the data were collected in two moments: In the first, the questionnaires were delivered inside an envelope and in the second the terms were filled with the applicator in order to avoid further constraints. At the end of the study, it was possible to conclude that the highest prevalence of stress symptoms occurred in professionals of the physiotherapists, female, with less than 38 years and less than 4 years of profession. The data also reveal that, of the 55 individuals surveyed, 16 (29\%) presented positive criteria for work stress, $1(2 \%)$ in phase 1 or alert or alarm, $13(24 \%)$ in phase 2 or struggle or resistance And 2 (3\%) in stage 3 or exhaustion or exhaustion. Regarding the symptoms of stress manifested:Muscle tension $(81 \%)$ - in the last 24 hours; problem with memory, forgetfulness $(81 \%)$ - in the last month; constant physical wear and tear $(81 \%)$, excessive tiredness (75\%) and daily anxiety or anxiety $(69 \%)$ - in the last 3 months.It is proposed to reflect on possible measures to improve working conditions and suggest more elaborate studies on the repercussions of stress with emphasis on the professional of Intensive Care Unit.

Keywords:Work stress. Professional exhaustion. Occupational stress. Health professionals. ICU.

\section{Introdução}

As novas formas de organização do trabalho ressaltam o desenvolvimento de um trabalhador com habilidades múltiplas, que deve ser capaz não apenas de antever problemas e desenvolver soluções alternativas, mas também de sugerir novas linhas de ação (ZILLE; BRAGA; MARQUES, 2008).

O trabalho, dependendo da forma que é vivido, pode gerar prazer ou sofrimento ao trabalhador. Fatores como ansiedade, depressão, grandes exigências, cumprimentos de tarefas em prazos sofríveis, desemprego, alto custo devida, problemas familiares, concorrência, baixos salários, insatisfação com o trabalho contribuem para que o trabalhador apresente o estresse ocupacional. Como consequência das crises o trabalhador pode ausentar-se do trabalho, sentir cansaço excessivo, dificuldade de concentração, pode ser oneroso executar tarefas e suas relações interpessoais podem ser afetadas (COSTA, 2006; LIPP; MALAGRIS, 2001).

Segundo Selye (1965) o estresse nada mais é do que um estado de alteração orgânica em resposta a qualquer sobrecarga dos recursos do corpo. Ele também descreve a síndrome de adaptação geral, que é a reação adaptativa do corpo quando submetido a agentes estressores. Ela é caracterizada pela existência de três fases: a) reação de alarme, assinalada pela queda da resistência e decorrente da ativação do sistema nervoso simpático em que o corpo fica pronto para enfrentar o desafio; b)resistência, na qual o corpo se mantém ativado, ainda que em grau menos intenso, de forma a manter seus recursos disponíveis para o embate; c)considerada de 
exaustão, cujo corpo é exigido a manter-se ativado por período mais longo do que aquele que consegue suportar; o organismo entra em exaustão e torna-se vulnerável, há queda na capacidade de pensar, lembrar e de agir,assim como da capacidade de resposta do sistema imunológico.

O ambiente de trabalho é um sistema no qual o ser humano está inserido e pode ser causador de estresse (PARREIRA, 2006).O hospital por si só se configura como um ambiente insalubre, árduo e de risco ocupacional para seus profissionais pelo fato destes entrarem em contato com materiais de alta periculosidade, grande volume de trabalho e presença de situações afetuosas e extremas, que causam elevado nível de tensão. O funcionamento total da organização também exige o regime de turnos e plantões, permitindo os duplos empregos e as longas jornadas de trabalho, que são práticas comuns desses profissionais da saúde, como forma de complementar os ganhos e que contribuem para a excessiva carga de trabalho associada a esse contexto (MONTEIRO et al., 2013).

O Burnout pode ser definido como uma deficiência emocional e comportamental que resulta da exposição a altos níveis de estresse ocupacional, e é uma combinação de três fatores: exaustão emocional, despersonalização e realização pessoal (DOMAGALA et al., 2015). Perfeccionismo e sensação de culpa são fatores de risco para burnout, que embora possa ser grave tem sido vista como uma síndrome de contexto social, portanto contagiosa. Além disso, está sendo mencionado como modismo por não ter uma definição padronizada e clara (VAN MOL et al., 2015).

As consequências do burnout podem ser deterioração das funções psicológicas e fisiológicas, distúrbios de funções cognitivas, somático e excitação fisiológica, redução da imunidade celular, baixa produtividade, absenteísmo, rotatividade do emprego, abuso de álcool ou drogas, e outros efeitos (DOMAGALA et al., 2015).

Os profissionais mais suscetíveis ao desgaste laboral, especialmente à burnout são os da área assistencial, em contato direto e constante com seus clientes na prestação do serviço, como profissionais de educação e saúde (RIBEIRO; BARBOSA; SOARES, 2015). O estresse entre os profissionais de saúde é justificado pela experiência de trabalhar com pessoas que estão doentes, chateadas e ansiosas com sua condição e complicações. Altas exigências no local de trabalho, pouco controle sobre os resultados do seu trabalho, idade entre 25 e 44 anos, sexo feminino, estado civil casado, número elevado de anos na prática, privação do sono, presença 
de dor nas costas e um efeito negativo da prática na vida familiar estão associados com a síndrome de Burnout (ŚLIWIŃSKIet al, 2014).

Os profissionais que trabalham em ambientes considerados críticos, como as unidades de tratamento intensivo (UTI), apresentam alta predisposição para serem acometidos pelo sofrimento psíquico, tendo em vista a complexidade das ações ali realizadas, o estresse gerado durante a sua realização e a ocorrência de morte de pacientes (GOMES et al., 2006).

Este trabalho tem como objetivo geral investigar os fatores associados ao estresse laboral em profissionais que trabalham em ambiente de Unidade de Terapia Intensiva e como objetivos específicos: Avaliar o nível de estresse dos participantes; investigar os sintomas de estresse manifestados pelos profissionais pesquisados; traçar o perfil sociodemográfico dos participantes; realizar uma análise comparativa em relação ao nível de estresse e os dados sociodemográficos.

Justifica-se por se tratar de uma condição laboral emergente e pelo ambiente das Unidades de Terapia Intensiva ser tão exigente e de alta tensão, além do que são escassos os estudos sobre estresse nos hospitais estudados e que contemplam a classe profissional dos fisioterapeutas, no Brasil. Segundo Van Mol et al. (2015) trabalhar em UTI é um desafio que demanda grande carga emocional, e o preço do cuidar pode se tornar um fardo para a vida pessoal dos profissionais, manifestando-se em fadiga ou síndrome de Burnout. Ainda segundo o autor a verdadeira magnitude do sofrimento emocional nos profissionais de saúde UTI permanece incerta devido a uma falta de pesquisas sobre o tema nas unidades.

A relevância da pesquisa baseia-se na contribuição dos resultados da pesquisa para os hospitais estudados, uma vez que poderão intervir positivamente no contexto laboral e até mesmo pessoal, melhorando a assistência ao paciente de UTI e a satisfação dos profissionais com seus trabalhos; e para a sociedade científica, podendo subsidiar outros trabalhos científicos na área buscando evidências que fundamentem uma melhor compreensão dos aspectos relacionados.

A seguinte questão de pesquisa foi abordada: Qual a relação do estresse do ambiente de UTI com o maior desgaste profissional? As hipóteses esperadas são: Profissionais submetidos a estresse laboral têm as relações inter e intrapessoais prejudicadas, o absenteísmo é um prejuízo decorrente do esgotamento profissional e as complicações mais sérias são a Síndrome de Burnout e a depressão. 


\section{Método}

Trata-se de um estudo transversal, observacional, descritivo e analítico de abordagem quantitativa.Esta pesquisa caracteriza-se quanto à abordagem como quantitativa. Para Dalfovo, Lana e Silveira (2008), no uso da abordagem quantitativa tudo pode ser mensurado em números, classificados e analisados utilizando-se técnicas estatísticas. Quanto aos objetivos se define como descritiva e exige do investigador uma série de informações sobre o que deseja pesquisar e pretende descrever os fatos e fenômenos de determinada realidade (TRIVIÑOS, 1987). E quanto aos procedimentos técnicos se configura como transversal, no qual os dados são coletados em um único momento do tempo buscando descrever o estado de uma ou mais variáveis naquele momento (SAMPIERI et al., 1991).

O estudo foi desenvolvido em dois hospitais localizados na cidade de Barbalha, Cariri cearense, Hospital do Coração do Cariri (HCC) e Hospital Santo Antônio (HSA) compreendendo o período entre agosto de 2016 a novembro de 2016. A escolha dessas instituições deveu-se por serem os hospitais de referência na cidade em questão e na região metropolitana do Cariri,pela facilidade de entrada na organização e disponibilidade de seus profissionais.

O trabalho foi realizado com profissionais de duas UTIs de hospitais de Barbalha, Ceará. A Unidade Coronariana do hospital do Coração do Cariri é composta por 13 médicos, 03 fisioterapeutas, 04 enfermeiros e 15 técnicos de enfermagem, totalizando 35 profissionais. $\mathrm{Na}$ UTI neurológica trabalham 09 médicos, 05 fisioterapeutas, 04 enfermeiros e 15 técnicos de enfermagem, somando mais 33 e totalizando, ao final, 68 profissionais. A pesquisa pretendeu abordar o maior número de profissionais possível entre os dias 31 de outubro a 11 de novembro de 2016, sendo aplicado de segunda a sexta-feira no período diurno, dependendo da disponibilidade do pesquisador e dos profissionais. Alguns profissionais foram excluídos da amostra por haver conflito de interesses ou não terem disponibilidade para responder o questionário. Todos os profissionais estão devidamente contratados e atuantes no hospital investigado. O tipo da amostra se deu por conveniência.

A seleção obedeceu aos seguintes critérios de inclusão:profissionais que trabalhem no setor de UTI da instituição com carga horária de pelo menos 12 horas/semanais; profissionais

528 Id on Line Rev. Mult. Psic. V.12, N. 42, p. 524-547, 2018 - ISSN 1981-1179 Edição eletrônica em http://idonline.emnuvens.com.br/id 
contratados pelo hospital e atuantes no período da coleta de dados. Foram excluídos da pesquisa os profissionais que apresentavam conflito de interesse com a pesquisa.

Foram aplicados dois instrumentos de pesquisa: O questionário sociodemográfico, que consta na primeira parte referente ao modelo teórico para explicar o estresse ocupacional em gerentes (MTEG), desenvolvido, testado e validado por Zille (2005) e O Inventário de Sintomas de Stress para adultos de Lipp (ISSL), que fornece uma medida objetiva da sintomatologia do estresse em jovens acima de 15 anos e adultos. Sua aplicação leva aproximadamente 10 minutos e pode ser realizada individualmente ou em grupos de até 20 pessoas. O Instrumento é formado por três quadros referentes às fases do estresse. O primeiro quadro, composto de 15 itens referese aos sintomas físicos ou psicológicos que a pessoa tenha experimentado nas últimas 24 horas. O segundo, composto de dez sintomas físicos e cinco psicológicos, está relacionado aos sintomas experimentados na última semana. E o terceiro quadro, composto de 12 sintomas físicos e 11 psicológicos, refere-se a sintomas experimentados no último mês. Alguns dos sintomas que aparecem no quadro 1 voltam a aparecer no quadro 3, mas com intensidade diferente. No total, o ISSL apresenta 37 itens de natureza somática e 19 psicológicas, sendo os sintomas muitas vezes repetidos, diferindo somente em sua intensidade e seriedade. A fase 3 (quase-exaustão) é diagnosticada na base da frequência dos itens assinalados na fase de resistência.

Para evitar constrangimento dos profissionais que se submeteram à pesquisa, os questionários foram entregues dentro de um envelope e os termos foram preenchidos junto ao aplicador, em dois momentos. Os questionários envelopados e devidamente lacrados foram depositados em caixas discriminadas pela classe profissional. Os participantes gozaram de privacidade ao responderem os questionários em uma sala fechada.

$\mathrm{Na}$ análise dos resultados, para os participantes que apresentaram mais de uma fase de estresse, foi considerada a fase mais avançada.

Após coleta dos dados, os resultados foram codificados, digitados e analisados sobre uma estrutura previamente programada, através do Microsoft Excel.

Este trabalho foi cadastrado na Plataforma Brasil e submetido ao Comitê de Ética em pesquisa com seres humanos da Estácio FMJ. Todos os profissionais que concordaram em participar da pesquisa receberam uma breve explicação sobre a pesquisa, depois leram o termo de Consentimento Livre e Esclarecido (TCLE) e ao final assinaram. A declaração de anuência 
foi obtida através dos responsáveis legais dos hospitais estudados. Este trabalho segue todos os princípios éticos estabelecidos pela lei 466/2012, que adota definições para estudos que envolvam seres humanos,incluindo o manejo de dados e informações dos participantes (CONSELHO NACIONAL DE SAÚDE, 2012).

\section{Resultados}

\section{Caracterização da amostra}

Foram aplicados questionários em 55 profissionais, divididos entre 11 médicos (20\%), 07 fisioterapeutas (13\%), 07 enfermeiros (13\%) e 30 técnicos de enfermagem (54\%), funcionários das Unidades de Terapia Intensiva dos hospitais do Coração do Cariri e Santo Antônio, da cidade de Barbalha, CE. A amostragem alcançou aproximadamente 80,8\% da população-alvo, estimada em 68 profissionais e foi caracterizada segundo classe profissional, gênero, idade e estado civil; curso de especialização; tempo de trabalho na instituição; número de horas semanais trabalhadas e trabalho aos finais de semana e feriados; hábito de tabagismo e uso de bebidas alcoólicas; condições gerais de saúde e horas de sono por dia.

Iniciando pelos aspectos relacionados com o estresse, um dos dados a salientar neste estudo refere-se a apenas 16 (29\%) participantes preencheram os critérios para classificá-lo em alguma fase de estresse, segundo o Inventário de Sintomas de Estresse para Adultos de Lipp. $\mathrm{Na}$ prática, isto significa que a maioria dos profissionais tem uma percepção baixa das exigências que lhes são colocadas. A tabela 1 mostra o diagnóstico e o nível de estresse ocupacional para a amostra estudada.

Tabela 1 - Análise do nível de estresse da amostra pesquisada

\begin{tabular}{llllll}
\hline & Sem estresse & Alerta & Luta & Exaustão & Total \\
\hline $\mathbf{n}$ & 39 & 1 & 13 & 2 & 55 \\
\hline \% & 71 & 2 & 24 & 3 & 100
\end{tabular}

Fonte: Pesquisa direta (2016). 
Dos profissionais que exibiram níveis de estresse, 13 (24\%) encontram-se na fase de luta ou resistência, 2 (3\%) estão na fase de exaustão ou esgotamento e 1 (2\%) está na fase de alerta ou alarme.

O fato de haver predominância de pessoas na fase de resistência pode sugerir que os eventos estressores ainda se mantêm e o organismo procura restabelecer o equilíbrio procurando adaptar-se (COSTA, 2006). Segundo Lipp e Malagris (2001) a fase de resistência é o prolongamento dos sintomas de estresse da fase de alerta, em que a pessoa se sente motivada a realizar novos projetos e seu nível de produtividade é consideravelmente alto, mas o organismo não consegue se manter por muito tempo.

\section{Categoria, gênero, idade, estado civil e nacionalidade}

Do total, $58 \%$ é do sexo feminino e $42 \%$ do sexo masculino. Resultado de um processo histórico na prática do cuidar associado à evolução na participação feminina no mercado de trabalho, as mulheres são maioria no exercício das profissões na área da saúde (METZKER,2011).

A média de idade encontrada foi de 34,15 anos, variando de 19 a 65 anos. Um dos profissionais que respondeu o questionário não preencheu o campo idade, sendo a análise deste dado realizada com apenas 54 respondedores. Os profissionais com idade entre 29 e 38 anos de idade somaram 29 (54\%) sendo, portanto, a maioria, seguidos por aqueles com 39 a 48 anos totalizando 10 profissionais e correspondendo a $18 \%$. Duas pessoas tinham 49 a 58 anos (4\%) e apenas uma mais que 58 anos (2\%).

A distribuição da amostra segundo o estado civil revela maior proporção de casados (62\%), com menor parcela de solteiros (25\%), outros (9\%) e viúvos (4\%).

Apenas 1 entrevistado é de nacionalidade boliviana e 54 de nacionalidade brasileira. 
Tabela 2: Caracterização da amostra segundo categoria, gênero, idade, estado civil e nacionalidade

\begin{tabular}{llllll} 
Variável & n & \% & Variável & n & \% \\
\hline Classe & & & Sexo & & \\
\hline Médicos & 11 & 20 & Masculino & 23 & 42 \\
\hline Fisioterapeutas & 7 & 13 & Feminino & 32 & 58 \\
\hline Enfermeiros & 7 & 13 & & & \\
\hline Técnicos de & 30 & 54 & & & \\
Enfermagem & & & & &
\end{tabular}

\begin{tabular}{lll}
\hline Idade & & \\
\hline $19-28$ & 12 & 22 \\
\hline $29-38$ & 29 & 54 \\
\hline $39-48$ & 10 & 18 \\
\hline $49-58$ & 2 & 4 \\
\hline$>58$ & 1 & 2 \\
\hline Nacionalidade & & \\
\hline Brasileira & 54 & 98 \\
\hline Boliviana & 1 & 2 \\
\hline
\end{tabular}

Fonte: Pesquisa direta (2016).

\section{Tempo de trabalho na instituição}

Em relação ao tempo que o profissional atua na empresa pesquisada, a amostra se distribuiu da seguinte forma: 23 (43\%) exercem a função há mais de 6 anos; 22 (41\%) exercem a função de um a três anos; 05 (9\%) trabalham na instituição há menos de 1 ano e 04 (7\%) de 4 a 6 anos.Tais dados revelam que a organização tem no seu quadro de pessoal a maioria de profissionais que desempenham suas atividades há mais tempo na instituição.

Tabela 3 - Caracterização da amostra segundo tempo de trabalho

\begin{tabular}{lll}
\hline Tempo de trabalho & N & \% \\
\hline Menos de 1 ano & 5 & 9 \\
\hline De 1 a 3 anos & 22 & 41 \\
\hline De 4 a 6 anos & 4 & 7 \\
\hline Mais de 6 anos & 23 & 43 \\
\hline
\end{tabular}

Fonte: Pesquisa direta (2016). 


\section{Número de horas trabalhadas e trabalho aos sábados, domingos e feriados}

Segundo o art. $8^{\circ}$ da Lei n ${ }^{\circ}$ 3.999/1961 a duração normal do trabalho dos médicos, salvo acordo escrito, será de, no mínimo, 2 horas e, no máximo, 4 horas diárias. Aos médicos e auxiliares que contratarem com mais de um empregador, não é permitido o trabalho além de 6 horas diárias (PALÁCIO DO PLANALTO, 1961). A jornada de trabalho dos funcionários do Conselho Federal de Enfermagem é de 40 horas semanais, sendo 08 horas diárias, de segunda a sexta-feira. A lei $n^{\circ} 8.856 / 94$, art. $1^{\circ}$ afirma que os profissionais Fisioterapeuta e Terapeuta Ocupacional ficarão sujeitos à prestação máxima de 30 horas semanais de trabalho. Estas informações vão de encontro aos resultados desta pesquisa: Os profissionais das referentes instituições trabalham, em sua maioria, mais de 40 horas semanais $(n=33,62 \%)$, caracterizando excesso de trabalho. 9 (17\%) profissionais trabalham entre 20 e 30 horas, $8(15 \%)$ trabalham entre 30 e 40 horas e $3(6 \%)$ entre 12 e 20 horas .2 pessoas não responderam a este item. Lautert, Chaves e Moura (1999) afirma que se por um lado o excesso de trabalhos reduz as oportunidades de apoio social do indivíduo, causando insatisfação, tensão e outros problemas de saúde, por outro lado, a falta de trabalho pode causar sensação de tédio ao indivíduo.

Em relação ao trabalho realizado aos sábados, domingos e feriados, deve-se levar em conta que as horas trabalhadas nesses dias já foram consideradas no número total de horas trabalhadas. Dezoito (18) profissionais não responderam a este item, uma vez que esta questão só deveria ser respondida caso estes dias (sábados, domingos e feriados) não fizessem parte da jornada de trabalho. Considerando que $21(57 \%)$ profissionais realizam às vezes e $15(40 \%)$ realizam sempre algum trabalho aos sábados, domingos e feriados, fica explicado o fato de $62 \%$ dos pesquisados afirmarem ter carga horária semanal superior a 40 horas, mesmo, no entanto, o trabalho aos finais de semana fazer parte do assinalado no contrato de trabalho dos profissionais estudados. 
Tabela 4 - Caracterização da amostra segundo carga horária e trabalho aos finais de semana e feriados

\begin{tabular}{llllll}
\hline $\begin{array}{l}\text { Número de horas } \\
\text { trabalhadas }\end{array}$ & $\mathbf{N}$ & $\mathbf{\%}$ & $\begin{array}{l}\text { Trabalho aos } \\
\text { sábados, domingos e } \\
\text { feriados n=37 }\end{array}$ & $\mathbf{n}$ & $\%$ \\
\hline 12 a 20 & 3 & 6 & Sempre & 15 & 40 \\
\hline Mais de 20 a 30 & 9 & 17 & Às vezes & 21 & 57 \\
\hline Mais de 30 a 40 & 8 & 15 & $\begin{array}{l}\text { Raramente ou } \\
\text { nunca }\end{array}$ & 1 & 3 \\
\hline Mais de 40 & 33 & 62 & & & \\
\hline
\end{tabular}

Fonte: Pesquisa direta (2016)

\section{Curso de especialização}

Dos pesquisados 37 (67\%) relataram não ter nenhum curso de especialização, enquanto 4 (7\%) afirmaram ter curso de especialização incompleto, 12 (22\%) curso de especialização completo e 2 (4\%) mestrado incompleto. Ninguém respondeu mestrado completo e Ph.D.Todos os técnicos de enfermagem não possuem nenhum curso de especialização. Dentre os demais as áreas destacadas são UTI, urgência e emergência e medicina intensiva, dentre outras. $\mathrm{O}$ alto percentual de profissionais com especialização em cuidado em Unidade de Terapia Intensiva pode ser explicado pelo fato de os cuidados de terapia intensiva serem de maior complexidade e de os pacientes serem mais graves.

Tabela 5: Caracterização da amostra segundo curso de especialização

\begin{tabular}{lll}
\hline Curso de especialização & n & \% \\
\hline Nenhum & 37 & 67 \\
\hline Especialização Incompleta & 4 & 7 \\
\hline Especialização completa & 12 & 22 \\
\hline Mestrado incompleto & 2 & 4
\end{tabular}

Fonte: Pesquisa direta (2016).

Tabagismo e hábito de consumo de bebida alcoólica

No que diz respeito ao tabagismo, 53 (96\%) relataram não fazer uso de fumo até a data da pesquisa, enquanto $2(4 \%)$ afirmaram fazer uso. No que se refere à frequência com que cada 
indivíduo tem fumado atualmente todos dizem fazer uso menos que de costume. Quanto ao uso de bebidas alcoólicas, do total da amostra pesquisada, 32 (58\%) afirmam não consumir e 23 (42\%) disseram fazer uso. Dos que relataram fazer uso de alguma bebida alcoólica, 16 (69\%) disseram consumir uma a cinco unidades por semana; 5 (22\%)6 a 15 unidades por semana; e 2 (9\%), 16 a 35 unidades por semana. No que se refere à frequência com que cada indivíduo tem bebido atualmente, dentre o que fazem uso do álcool,13 (57\%) têm consumido menos que de costume, ao passo que 9 (39\%) têm consumido o mesmo de costume e 01 (4\%) mais do que de costume.

Tabela 6 - Caracterização da amostra segundo hábitos de tabagismo e alcoolismo

\begin{tabular}{lcclcc}
\hline Tabagismo & $\mathbf{n}$ & $\mathbf{\%}$ & $\begin{array}{l}\text { Frequência de } \\
\text { tabagismo }\end{array}$ & $\mathbf{n}$ & $\%$ \\
\hline Sim & 2 & 4 & $\begin{array}{l}\text { Mais que de } \\
\text { costume }\end{array}$ & 0 & 0 \\
\hline Não & 53 & 96 & $\begin{array}{l}\text { Mesmo que de } \\
\text { costume }\end{array}$ & 0 & 0 \\
\hline & & $\begin{array}{l}\text { Menos que de } \\
\text { costume }\end{array}$ & 2 & 100 \\
\hline Alcoolismo & 23 & 42 & Unidades por semana & \\
\hline Sim & 32 & 58 & 1 a 5 & 16 & 69 \\
\hline Não & & & 6 a 15 & 5 & 22 \\
\hline & & & 16 a 35 & 2 & 9 \\
\hline & & & Mais de 35 & 0 & 0 \\
\hline
\end{tabular}

\begin{tabular}{lll}
\hline $\begin{array}{l}\text { Frequência de } \\
\text { alcoolismo }\end{array}$ & & \\
\hline Mais que de costume & 1 & 4 \\
\hline $\begin{array}{l}\text { Mesmo que de } \\
\text { costume }\end{array}$ & 9 & 39 \\
\hline $\begin{array}{l}\text { Menos que de } \\
\text { costume }\end{array}$ & 13 & 57 \\
\hline
\end{tabular}

Fonte: Pesquisa direta (2016).

\section{Condições de saúde e quantidade de horas de sono por dia}

Dentre os profissionais avaliados $44(80 \%)$ nãoapresentam problemas de saúde, sendo que os outros 11(20\%) têm algum tipo de problema de saúde, destacando-se diabetes mellitus e gastrite (5\% cada), sobrepeso, HPV (Papiloma Vírus Humano), tireoideopatias, hipertensão 
arterial e nefrolitíase ( $2 \%$ cada). Uma taxa relativa a $2 \%$ da amostra afirmou fazer uso de qualquer medicação ansiolítica ou antidepressiva e $100 \%$ nunca apresentou infarto agudo do miocárdio (IAM). Em relação às situações estressantes no trabalho de Grazziano (2008) afirma que o estresse ocupacional pode acarretar no desenvolvimento de várias patologias como a hipertensão arterial, doença coronariana, além de distúrbios emocionais e psicológicos, como a ansiedade, depressão e baixa autoestima, trazendo repercussão negativa direta no desempenho e organização da empresa. $\mathrm{Na}$ área da saúde, especialmente, é possível verificar uma variedade de doenças que podem ser desencadeadas a partir de um stress excessivo e estas são decorrentes das alterações psicofisiológicas que ocorrem, no entanto são dependentes de predisposições individuais (LIPP;MALAGRIS, 2001).

Hanzelmann e Passos (2010) dissertam que o estresse interfere tanto o desempenho profissional quanto a vida particular, pois ele influencia todas as atividades realizadas pelo indivíduo. No entanto, a maneira com que a pessoa se enfrenta os fatores estressores definirá o estado de estresse ao qual se está sendo sujeitada e que modificações são provocadas por ele.

Em relação ao número de horas de sono por dia, os resultados ressaltam percentual mais alto para aqueles que dormem quatro a seis horas de sono por dia 20 (36\%), seguida por seis a oito horas 19(35\%). Com tempo de sono inferior a quatro horas/dia aparecem 10 pesquisados ou 18\% do total da amostra, dado que apresenta coerência com o número de profissionais que relataram insônia como um problema de saúde, tanto nas últimas 24 horas como nos últimos 3 meses.

Tabela 7 - Caracterização da amostra segundo condições de saúde e horas de sono

\begin{tabular}{llllll}
\hline $\begin{array}{l}\text { Problema } \\
\text { relacionado a saúde }\end{array}$ & $\mathbf{n}$ & $\mathbf{\%}$ & $\begin{array}{l}\text { Uso de ansiolítico ou } \\
\text { antidepressivo }\end{array}$ & $\mathbf{n}$ & $\%$ \\
\hline Sem patologias & 44 & 80 & Sim & 1 & 2 \\
\hline Diabetes & 3 & 5 & Não & 54 & 98 \\
\hline Gastrite & 3 & 5 & & & \\
\hline Sobrepeso & 1 & 2 & Infarto cardíaco & & \\
\hline Hipertensão arterial & 1 & 2 & Sim & 0 & 0 \\
\hline Tireoideopatias & 1 & 2 & Não & 55 & 100 \\
\hline HPV & 1 & 2 & & & \\
\hline Nefrolitíase & 1 & 2 & & & \\
\hline
\end{tabular}

\begin{aligned} & \hline Número de horas de sono por dia \\ & \hline 536 $\begin{array}{l}\text { Id on Line Rev. Mult. Psic. V.12, N. 42, p. 524-547, 2018 - ISSN 1981-1179 } \\ \text { Ediçăo eletrônica em http://idonline.emnuvens.com.brlid }\end{array}\end{aligned}$

\section{Hobbie}




\begin{tabular}{llllll}
\hline Até 4 & 10 & 18 & Sim & 25 & 45 \\
\hline Mais de 4 a 6 & 20 & 36 & Não & 30 & 55 \\
\hline Mais de 6 a 8 & 19 & 35 & & & \\
\hline Mais de 8 & 6 & 11 & & & \\
\hline
\end{tabular}

Fonte: Pesquisa direta (2016).

Tabela 8 -Sintomas relatados pela amostra com critérios para estresse

Continua

\begin{tabular}{lll}
\hline Sintomas nas últimas 24 horas & n & \% \\
\hline Mãos e/ou pés frios & 1 & 6 \\
\hline Boca seca & 4 & 25 \\
\hline Nó ou dor no estômago & 6 & 37 \\
\hline Aumento da sudorese & 0 & 0 \\
\hline Tensão muscular & 13 & 81 \\
\hline Aperto na mandíbula/ranger dos dentes & 2 & 12 \\
\hline Diarreia passageira & 1 & 6 \\
\hline Insônia, dificuldade para dormir & 8 & 50 \\
\hline Taquicardia & 3 & 19 \\
\hline Respiração ofegante, entrecortada & 3 & 19 \\
\hline Hipertensão súbita e passageira & 0 & 0 \\
\hline Mudança de apetite (muito ou pouco) & 9 & 56 \\
\hline Aumento súbito de motivação & 1 & 6 \\
\hline Entusiasmo súbito & 0 & 0 \\
\hline Vontade súbita de novos projetos & 2 & 12 \\
\hline Sintomas no último mês & & \\
\hline Problema com a memória esquecimento & 13 & 81 \\
\hline Mal estar generalizado, sem causa & 7 & 44 \\
\hline Formigamento de extremidades & 3 & 19 \\
\hline Sensação de desgaste físico constante & 13 & 81 \\
\hline Mudança de apetite & 8 & 50 \\
\hline Surgimento de problemas & 4 & 24 \\
\hline dermatológicos & & \\
\hline Hipertensão arterial confirmada & 0 & 0 \\
\hline Cansaço constante & 9 & 56 \\
\hline Gastrite prolongada & 5 & 31 \\
\hline Tontura, sensação de estar flutuando & 3 & 19 \\
\hline Sensibilidade emotiva excessiva & 4 & 24 \\
\hline Dúvidas quanto a si próprio & 6 & 37 \\
\hline
\end{tabular}




\begin{tabular}{|c|c|c|}
\hline Pensamento sobre um só assunto & 5 & 31 \\
\hline Irritabilidade excessiva & 8 & 50 \\
\hline Diminuição da libido & 2 & 12 \\
\hline Sintomas nos últimos 3 meses & $\mathbf{n}$ & $\%$ \\
\hline Diarreias frequentes & 0 & 0 \\
\hline Dificuldades sexuais & 1 & 6 \\
\hline Formigamento de extremidades & 1 & 6 \\
\hline Insônia & 8 & 50 \\
\hline Tiques nervosos & 4 & 24 \\
\hline Hipertensão arterial confirmada & 0 & 0 \\
\hline Problemas dermatológicos prolongado & 2 & 12 \\
\hline Mudança extrema de apetite & 6 & 37 \\
\hline Taquicardia & 3 & 19 \\
\hline Tontura frequente & 3 & 19 \\
\hline Úlcera & 0 & 0 \\
\hline Impossibilidade de trabalhar & 0 & 0 \\
\hline Pesadelos & 4 & 24 \\
\hline $\begin{array}{l}\text { Sensação de incompetência em todas as } \\
\text { áreas }\end{array}$ & 1 & 6 \\
\hline Vontade de fugir de tudo & 4 & 24 \\
\hline Apatia, vontade de nada fazer, depressão & 1 & 6 \\
\hline Cansaço excessivo & 12 & 75 \\
\hline $\begin{array}{l}\text { Pensamento constante no mesmo } \\
\text { assunto }\end{array}$ & 6 & 37 \\
\hline Irritabilidade sem causa aparente & 7 & 44 \\
\hline Angústia ou ansiedade diária & 11 & 69 \\
\hline Hipersensibilidade emotiva & 6 & 37 \\
\hline Perda do senso de humor & 7 & 44 \\
\hline
\end{tabular}

Fonte: Pesquisa direta (2016).

\section{Discussão}

\section{Avaliação do nível de estresse}

Para a interpretação e análise do nível de intensidade do estresse laborativo da amostra estudada utilizou-se como referência o Inventário de Sintomas de Stress para adultos de Lipp (ISSL), validado por Lipp e Guevara no ano de 1994. Este questionário pretende avaliar se o 
participante possui algum sintoma de estresse ou está propenso a este e segue os seguintes critérios: ausência de estresse, alerta ou alarme, resistência ou luta e exaustão ou esgotamento. Para os participantes que apresentaram mais de uma fase de estresse, foi considerada a fase mais avançada.

A reação de alarme é assinalada pela queda da resistência e decorrente da ativação do sistema nervoso simpático em que o corpo fica pronto para enfrentar o desafio. Na fase de resistência o corpo se mantém ativado, ainda que em grau menos intenso, de forma a manter seus recursos disponíveis para o embate; e na exaustão, o corpo é exigido a manter-se ativado por período mais longo do que aquele que consegue suportar; o organismo entra em exaustão e torna-se vulnerável, há queda na capacidade de pensar, lembrar e de agir, assim como da capacidade de resposta do sistema imunológico (SELYE, 1965).

O indivíduo na fase 1 é mulher, casada, que trabalha na função há mais de 6 anos e que dorme de 4 a 6 horas por dia. Dentre os profissionais que estão na fase 2, que são a maioria que apresenta sintomatologia de estresse, predominam com pouca vantagem as mulheres, os casados, na faixa etária de 29 a 38 anos, que trabalham de 1 a 3 anos na função, sem curso de especialização, que dormem 4 a 6 horas por dia e tem hobbie. Daquelas que estão na fase 3 , todas são mulheres, solteiras ou em outro estado civil que não casadas ou viúvas, que trabalham entre 1 e 6 anos na função, sem curso de especialização e tempo de sono de mais de 4 a 6 horas por dia.

\section{Relação das variáveis sociodemográficas com o nível de estresse}

Percebe-se neste estudo, que 3 dos 11 médicos, 3 dos 7 fisioterapeutas, 1 dos 7 enfermeiros e 9 dos 30 técnicos estão sob efeito do estresse no ambiente de trabalho. Na área da saúde é possível verificar uma variedade de doenças que podem ser desencadeadas a partir de um stress excessivo (LIPP; MALAGRIS, 2001). Consequentemente, estas doenças podem provocar problemas na área profissional, como absenteísmo, atrasos, desempenho insatisfatório, queda de produtividade e problemas de relacionamento (FONTANA, 1994; SANTOS; ROCHA, 2003). Ao correlacionar o número de profissionais estressados pelo total de cada classe, pode-se concluir que os fisioterapeutas compõem a classe trabalhista que mais 
sofre com o desgaste laboral, seguida pelos técnicos de enfermagem, depois pelos médicos e por fim, pelos enfermeiros. Em uma pesquisa para avaliar o nível de estresse dos profissionais de saúde, Carvalho e Malagris (2007) dissertam que dentre as profissões de saúde, as que apresentaram maior índice de estresse laboral são as de serviço social, enfermagem e medicina, confrontando os resultados obtidos nesta pesquisa.

No geral, as participantes do sexo feminino obtiveram uma média de estresse mais elevada que os do sexo masculino, enquanto elas somaram $34,37 \%$ eles obtiveram $21,74 \%$ de sintomas que caracterizam alguma fase de estresse segundo o Inventário de Sintomas de Stress para adultos de Lipp (ISSL). Entre as mulheres, a maioria está na fase de luta (n=8, 25\%), assim como também todos os homens com estresse ( $\mathrm{n}=5,22 \%$ do total da amostra masculina). $6 \%$ (2) das mulheres estão na fase de exaustão e 3\% (1) na fase de alerta. França e Rodrigues (2002) comentam que os sintomas de estresse na mulher se dão pelos múltiplos papéis que a mulher desempenha tanto no mercado de trabalho quanto na qualidade de mãe, esposa e do lar, necessitando, desta maneira, conquistar um lugar merecido em vários espaços durante o decorrer da vida. Tais resultados também confirmam os dados obtidos por Gomes et al. (2009) em pesquisas realizadas com enfermeiros portugueses, onde a diferença de sexo colocou as mulheres com mais problemas de estresse e com mais dificuldades de saúde física que os homens.Outros trabalhos, como os realizados por Moraes et al. (2001) e Lipp e Tanganelli (2002) confirmam a maior predisposição do sexo feminino a estados de estresse.

Quando se estratificou por faixa etária, verificou-se maior percentual de estresse nas faixas etárias entre 29 e 38 anos $(n=7,44 \%)$ e 19 a 28 anos $(n=6,37 \%)$, seguidos dos com idade entre 39 e 48 anos $(n=2,13 \%)$ e entre 49 e 58 anos $(n=1,6 \%)$, os demais não contemplaram a classificação de estresse. Ressalta-se aqui o grupo de profissionais jovens com maior nível de estresse, corroborando com os trabalhos de Gomes et al. (2009), que afirma que este grupo relata experiências mais estressantes no trabalho devido a excesso de trabalho e insatisfação com a remuneração. Em seus estudos Gurrer e Bianchi (2008) verificaram resultados semelhantes, nos quais enfermeiros com idade entre 31 e 40 anos obtiveram maior nível de estresse em relação com os com 41 a 50 anos; e estes se mostraram mais estressados que os com mais de 51 anos.

Realizando-se a relação entre os profissionais estressados e totais, os casados mostraram-se menos estressados que aqueles com outro tipo de estado civil, os solteiros vêm 
logo em seguida, seguidos por viúvos, sendo os que estão em outro estado civil ficando em primeiro lugar entre os mais estressados. Os resultados desta pesquisam vão ao encontro dos trabalhos de Wurm et al. (2016) realizados com médicos austríacos mostram que médicos casados têm um menor risco de escores de depressão moderada ou grave em comparação com os seus colegas solteiros. A pesquisa de Gomes et al. (2008) com profissionais na área da saúde, demonstrou uma maior tendência dos solteiros para relatarem maior stress relacionado com a instabilidade profissional na carreira. Na literatura internacional, também existem algumas indicações sobre a possibilidade de os solteiros serem mais sensíveis a problemas de stress ocupacional do que os casados e os profissionais divorciados (MASLACH; SCHAUFELI; LEITER, 2001), divergindo dos resultados desta pesquisa, que mostrou solteiros e viúvos em situação intermediária de estresse, e a maior prevalência em outras situações de estado civil. Em relação a gênero a satisfação com o casamento, filhos, trabalho, relações interpessoais, bem como a sexualidade pode contribuir para a redução do desgaste entre os homens. As mulheres que estão satisfeitos com seus filhos, família, saúde, tempo livre e relações interpessoais são menos propensas a burnout. A deterioração financeira entre as mulheres e a falta de tempo livre entre os homens podem induzir burnout. (ŚLIWIŃSKIet al., 2014).

No que se refere ao tempo de trabalho na instituição, é possível verificar que os profissionais com critérios para estresse concentram-se divididos nas faixas de 1 a 3 anos ( $n=6$, $37 \%)$ e mais que a 6 anos de trabalho $(n=5,31 \%)$. Os que trabalham há menos de 1 ano $(n=3$, $19 \%)$ e entre 4 e 6 anos $(n=2,13 \%)$ seguem atrás. Os mais inexperientes, com tempo de trabalho inferior a 4 anos seguem liderando as taxas de estresse laboral. Isto pode estar relacionado à instabilidade profissional e na carreira. Em termos gerais, estes tipos de problemas também foram evidentes num estudo realizado por Gomes et al. (2009) com profissionais da área da saúde, onde foram precisamente os mais novos e inexperientes os que descreveram mais queixas relativas ao salário e à instabilidade laboral. Em relação ao número de horas trabalhadas, os mais estressados são os que trabalham mais de 20 a 30 horas semanais e a maioria encontra-se na fase de luta. Da mesma forma, aqueles que sempre trabalham aos finais de semana e feriados, não fazendo parte de sua carga horária habitual, relataram maior estresse.

Os mais estressados são os que não possuem nenhum curso de especialização $(n=10$, que corresponde a $18 \%$ de toda a amostra). Como nenhum dos técnicos de enfermagem possui 
especialização e essa classe se revelou com muitos sintomas relativos ao estresse, pode haver associação inversamente proporcional de maior nível de estresse e especialização.

No que diz respeito ao tabagismo, dos 2 profissionais que fumam, 1 tem sintomatologia para estresse e está na fase de luta. A relação entre consumo de bebida alcoólica e nível de estresse apresentado pelos profissionais pesquisados, pode ser verificada de modo que 11 (48\%) do total dos que fazem uso de bebida alcoólica, estarem em alguma fase de estresse. Nas pesquisas de Metzker (2011),com base nos testes de inferência estatística, há relação de dependência direta entre o consumo de bebida alcoólica e nível de estresse para os profissionais avaliados $(\mathrm{p}<0,05)$. Do total dos respondedores com critérios positivos para estresse e que fazem uso de alcoólica ( $\mathrm{n}=11), 9$ (82\%) fazem uso de 1 a 5 unidades, 1 (9\%) de 6 a 15 e 1 (9\%) de 16 a 35. Além disso, 7 (64\%) relatam frequência de consumo menor que de costume, 3 (27\%) o mesmo que de costume e apenas 1 (9\%) mais do que de costume. Para Costa (2006) muitos profissionais estressados podem adoecer pelo uso excessivo de álcool ou fumo como uma "válvula de escape".

É possível verificar que dos $16(100 \%)$ profissionais com critérios positivos para estresse, em relação a problemas de saúde, 13 (81\%) não manifestaram problema de saúde e 3 (19\%) tiveram identificado algum problema de saúde. Da mesma maneira, dos profissionais sem estresse $(n=39) 79 \%$ não apresenta problema relacionado à saúde. Na pesquisa de Metzker (2011), foi possível afirmar que não há direta relação de dependência entre o nível de estresse apresentado pelos profissionais pesquisados e a existência de algum problema de saúde $(\mathrm{P}>0,05)$, sugerindo que os problemas de saúde dos profissionais avaliados não são decorrentes do estresse ocupacional. Segundo Rangé (2001), as doenças que parecem estar relacionadas com o estresse seriam: a hipertensão arterial, câncer, vitiligo, lúpus, doenças do trato gastrointestinal, do trato respiratório e doenças relacionadas à imunidade, entre outras, coincidindo com os resultados encontrados nesta pesquisa.

Considerando os profissionais que tiveram critérios positivos para estresse, a amostra se distribuiu da seguinte forma no que concerne a número de horas de sono por dia: um $(6 \%)$ indivíduo dormia até quatro horas/dia, 11 (65\%) dormiam quatro a seis horas de sono/dia, 3 (17\%) disseram dormir seis a oito horas/dia e 2 (12\%) mais de 8 horas. Com tempo de sono inferior a quatro horas/dia aparece um pesquisado ou $6 \%$ do total da amostra com sintomas de 
estresse, dado que apresenta incoerência com o número de profissionais que relataram insônia como um problema de saúde (50\%).

Em relação a hobbie, dos $16(100 \%)$ daqueles com fatores positivos para estresse, 10 têm algum tipo de atividade de lazer e 6 não têm.

\section{Sinais e sintomas}

No tocante aos sintomas relatados pelos profissionais que responderam o questionário de Lipp, a tabela 04 representa a distribuição dos sintomas nas últimas 24 horas, no último mês e nos últimos 3 meses para a amostra estudada.

Os principais sintomas assinalados pelos indivíduos com critérios positivos para estresse $(\mathrm{n}=16)$ foram: Tensão muscular $(81 \%)$ - nas últimas 24 horas;problema com a memória, esquecimento (81\%) e sensação de desgaste físico constante( $81 \%)$ - no último mês;cansaço excessivo (75\%) e angústia ou ansiedade diária (69\%) - nos últimos 3 meses.

Os sintomas obtidos no trabalho desenvolvido por Metzker (2011) estão em consonância com esta pesquisa. Este autor verifica que fadiga e tensão muscular correspondem ao construto secundário aumento de tônus muscular, tontura e/ou vertigem, falta ou excesso de apetite e relaxamento; assim como a ansiedade diz respeito ao construto sintomas psíquicos, decorrentes dos sistemas nervoso simpático e gástrico.

\section{Considerações Finais}

O presente estudo abordou o estresse laboral em profissionais que trabalham em ambiente de Unidade de Terapia Intensiva, investigando os fatores associados ao estresse em relação às variáveis sociodemográficas e ocupacionais,com o intuito de identificar a prevalência de estresse na amostra estudada.

Analisando-se os profissionais diagnosticados com a síndrome, percebeu-se prevalência naqueles com classe fisioterapeuta, sexo feminino, idade menor do que 38 anos e ter menos de 4 anos de profissão.

543 Id on Line Rev. Mult. Psic. V.12, N. 42, p. 524-547, 2018 - ISSN 1981-1179 Edição eletrônica em http://idonline.emnuvens.com.br/id 
Como o estresse está sendo uma condição que vem emergindo no contexto laborativo e pode acarretar diversas patologias tanto psicológicas quanto físicas e intervir negativamente no contexto profissional, é necessária uma reflexão sobre possíveis medidas a serem adotadas no sentido de melhorar as condições de trabalho e reduzir os efeitos deletérios do estresse laboral, uma vez que estes profissionais são os responsáveis pela vida de pacientes graves, que necessitam de atenção integrada e continuada e muitas vezes estão em risco de óbito.

É importante destacar que os resultados desta pesquisa limitam-se à instituição estudada, apesar de alguns dados representarem também a realidade mundial. Sugere-se a realização de estudos mais elaborados sobre as repercussões do estresse com ênfase no profissional de UTI devido à escassez de estudos encontrados na literatura sugerimos e pelo tema possuir grande relevância para a saúde pública.

\section{Referências}

CARVALHO, L.; MALAGRIS, L.E.N. Avaliação do nível de stress em profissionais de saúde. Estudos e pesquisas em psicologia, Rio de Janeiro, UERJ, v. 7, n. 3, p. 570-582, 2007. Disponível em: <http://www.revispsi.uerj.br/v7n3/artigos/pdf/v7n3a16.pdf>. Acesso em: 26 out. 2016.

BRASIL. Decisão n ${ }^{\circ}$ 154/2016. Estabelece procedimentos para jornada de trabalho, controle de frequência e banco de horas no âmbito do Cofen. Diário Oficial da União. Disponível em: $<$ http://www.cofen.gov.br/decisao-cofen-no-01542016_41714.html >. Acessado em: 29 set. 2016.

CONSELHO NACIONAL DE SAÚDE. Resolução n 466, de 12 dedezembro de 2012. Diário Oficial da União. Disponível em: <http://conselho.saude.gov.br/resolucoes/2012/Reso466.pdf>. Acesso em 12 set. 2016.

CONSELHO FEDERAL DE FISIOTERAPIA E TERAPIA OCUPACIONAL. COFFITO. Enfim, a jornada máxima de 30 horas. O COFFITO. São Paulo, ano 1, n. 1, jan. 1995.

COSTA, Denize Dalla. O estresse do administrador de empresas privadas: um estudo em Cascavel - PR. 2006. 104 f. Dissertação (Mestrado em Psicologia), Universidade Católica Dom Bosco (UCDB), Campo Grande, 2006.

DALFOVO, Michael Samir; LANA, Rogério Adilson; SILVEIRA, Amélia. Métodos quantitativos e qualitativos: um resgate teórico. Revista Interdisciplinar Científica Aplicada, Blumenau, v.2, n.4, p.01- 13, Sem II. 2008. 
DOMAGALA et al. The Interrelationships of Coping Styles and Professional Burnout Among Physiotherapists: A Cross-Sectional Study. Medicina (Baltimore); v. 94, n. 24: e906.Jun,2015.

FRANÇA, A. C. L.; RODRIGUES, A. L. Stress e trabalho: uma abordagem psicossomática. 3. ed. São Paulo: Atlas, 2002.

FONTANA, D. Estresse: faça dele um aliado e exercite a autodefesa. São Paulo: Saraiva, 1994.

GOMES, A. R. et al. (2008). Stresse, saúde física, satisfação e "burnout" em profissionais de saúde: análise das diferenças em função do sexo, estado civil e agregado familiar. In: M. G. Pereira, C. Simães \& T. McIntyre (Orgs.), Actas do II congresso família, saúde e doença: Modelos, investigação e prática em diferentes contextos de saúde ( $2^{\text {a }}$ ed., Vol. IV, pp. 178192). Braga: Universidade do Minho.

GOMES, A. R.; CRUZ, J. F.; CABANELAS, S. (2009). Estresse ocupacional em profissionais de saúde: um estudo com enfermeiros portugueses. Psicologia: Teoria e Pesquisa, v. 25, n. 3, p. 307-318.

GOMES, G. et al. O sofrimento psíquico em trabalhadores de UTI interferindo no seu modo de viver a enfermagem. Revista de Enfermagem, v. 14, n. 1, p. 93-99, 2006.

GRAZZIANO, E. S. Estratégia para redução do estress e burnout entre enfermeiroshospitalares. São Paulo, 2008. Disponível em: $<$ http://pandora.cisc.usp.br/teses/disponiveis/7/7139/tde-14052009 101907/publico/Eliane_Grazziano.pdf>. Acesso em: 15 nov. 2016.

GUERRER, Francine Jomara Lopes; BIANCHI, Estela Regina Ferraz. Caracterização do estresse nos enfermeiros de unidades de terapia intensive. Rev. Esc. Enferm. USP; v. 42, n. 2,p. 355-362, 2008.

HANZELMANN, R. S; PASSOS, J. P. Imagens e representações da enfermagem acerca do stress e sua influência na atividade laboral. Rev. esc. enferm. USP, São Paulo, v. 44, n. 3, 2010.

LAUTERT, Liana; CHAVES, Enaura H. B.; MOURA, Gisela M. S. S. O estresse na atividade gerencial do enfermeiro. Rev. Panam. Salud Publica/Pan Am J Public Health v. 6, n.6, 1999.

LIMA et al. Vulnerabilidade ao burnout entre médicos de hospital público do Recife. Ciênc. saúde coletiva, v. 18, n. 4, p.1051-1058, 2013.

LIPP, M. E. N.; Malagris, L. E. N. O stress emocional e seu tratamento. In B. Rangé (Org). Psicoterapias cognitivo-comportamentais: um diálogo com a psiquiatria (pp.475-490). Porto Alegre: Artmed, 2001. 
LIPP, M.E.N.; TANGANELLI, M.S. Stress ocupacional de magistrados da justiça do trabalho: diferenças entre homens e mulheres. Psicologia: Reflexão e Crítica, v. 15, p. 537-548, 2002.

MASLACH, C., SCHAUFELI, W. B., \& LEITER, M. P. Job burnout. Annual Review of Psychology, v. 52, p. 397-422, 2001.

METZKER, C.A.B. O FISIOTERAPEUTA E O ESTRESSE NO TRABALHO: estudo de caso em um hospital filantrópico da cidade de Belo Horizonte-MG. Pedro Leopoldo. Faculdades Integradas de Pedro Leopoldo, 2011.

MORAES, L.F.R. et al. Implicações do gênero na qualidade de vida e estresse no trabalho da policia militar do estado de Minas Gerais. In: V CONGRESSO DE CIÊNCIAS HUMANAS, LETRAS E ARTES DE OURO PRETO, Anais..., 2001.)

PALÁCIO DO PLANALTO. Lei no 3.999, de 15 de dezembro de 1961. Altera o saláriomínimo dos médicos e cirurgiões dentistas. Disponível em: <http://www.planalto.gov.br/ccivil_03/Leis/1950-1969/L3999.html>. Acesso em 29 set. 2016.

PARREIRA, G.S. Psicólogos e estresse: estudo de caso com profissionais atuantes em uma clínica credenciada pelo Detran-MG. Pedro Leopoldo-MG. 2006. [Dissertação de Mestrado Administração], Faculdades Integradas de Pedro Leopoldo, 2006.

RANGÉ, B. Psicoterapia comportamental. In: RANGÉ, B. (Org.). Psicoterapia comportamental e cognitiva: pesquisa, prática, aplicações e problemas. Capítulo 3. São Paulo: Livro Pleno, 2001.

RIBEIRO, L. C. C; BARBOSA, L. A. C. R; SOARES, A. S. EVALUATION PREVALENCE OF BURNOUT AMONG PUBLIC SCHOOL TEACHERS AND THEIR RELATIONSHIP TO SOCIODEMOGRAPHIC.R. Enferm. Cent. O. Min.; v. 5, n. 3, p. 1741-1751, set/dez 2015.

SAMPIERI, R.H. et al. Metodologia de la investigación. México, McGraw-Hill, 1991.

SANTOS, C. M. C.; ROCHA, L. S. A. D. O stress e o professor em uma escola de formaçãode professores na cidade do Rio de Janeiro. Monografia não-publicada apresentada ao Departamento de Psicologia Clínica, Instituto de Psicologia, Universidade Federal do Rio deJaneiro, 2003.

SELYE, H. Stress: a tensão da vida. 2. ed., 351 p. São Paulo: IBRASA, 1965.

SILVA, P.L.A. Percepção de fontes de estresse ocupacional, coping e resiliência no fisioterapeuta. [Dissertação de Mestrado em Fisioterapia]. Programa de Pós-Graduação em Ciências Ambientais e Saúde. Universidade Católica de Goiás.Goiânia, 2006. 
ŚLIWIŃSKI, Zbigniewet al.LIFE SATISFACTION AND RISK OF BURNOUT AMONG MEN AND WOMEN WORKING AS PHYSIOTHERAPISTS. International Journal of Occupational Medicine and Environmental Health;v. 27, n. 3, p. 400 - 412, 2014.

TRIVIÑOS, A. N. S. Introdução à pesquisa em ciências sociais: a pesquisa qualitativa em educação. São Paulo: Atlas, 1987.

WURM, Walter et al. "Depression-Burnout Overlap in Physicians." Ed. Jacobus van Wouwe. PLoS ONE, 11.3, 2016.

ZILLE, L. P. Novas perspectivas para abordagem do estresse ocupacional em gerente: estudos em organizações brasileiras de diversos setores.(Tese de Doutorado). Belo Horizonte: CEPEAD/FACE/UFMG, 2005.

ZILLE, P.L.; BRAGA, C.D.; MARQUES, A.L. Estresse no trabalho: estudo de caso com gerentes que atuam em uma instituição financeira nacional de grande porte. Revista de Ciências de Administração, v. 10, n. 21, p. 175-196, mai./ago, 2008.

\section{Como citar este artigo (Formato ABNT):}

MACÊDO, Antonio Taumaturgo Sampaio; SOUSA, Morgana Tavares Dantas; GOMES, Rodrigo Luís Mousinho; ROLIM, Marco Antônio Bezerra; BASTOS, Jussara Evila Pinheiro; DANTAS, Renata da Silva Adonias; BATISTA, Hermes Melo Teixeira; LEITE, Eliane de Sousa. Estresse Laboral em Profissionais da Saúde na Ambiência da Unidade de Terapia Intensiva. Id on Line Rev.Mult. Psic., 2018, vol.12, n.42, p. 524-547. ISSN: 1981-1179.

Recebido: 19/09/2018;

Aceito: 04/10/2018 